TRACE - Tree Rings in Archaeology, Climatology and Ecology, Vol. 7, pp 151-157.

Wang, Z. and M. M. Calderon and M. G. Carandang (2006): Effects of resin tapping on optimal rotation age of pine plantation. Journal of Forest Economics 11: 245-260.

Wu, H. X., M. B. Powell, J. L. Yang, M. Ivkovic and T. A. MCRAE (2007): Efficiency of early selection for rotationaged wood quality traits in radiate pine. Annals of Forest Science 64: 1-9.

XIE, C. Y. (2008): Ten-year results from red alder (Alnus rubra Bong.) provenance-progeny testing and their implications for genetic improvement. New Forests 36: 273-284.

XU, Y. B. (1994): The collected research works on masson pine in Guangdong province. Guangdong Higher Education Press (Ed 1). Guangzhou. pp 11-23.

YAMADA, Y. (1962): Genotype by environment interaction and genetic correlation of the same trait under different environments. Japanese Journal of Genetics 37: 498-509.
ZENG, L. H. and S. L. YUE (1984): Correlation between resin-yielding capacity and growth traits of Pinus massoniana. Subtropical Forestry Science and Technology 1: $16-18$.

ZHANG, B. W. (2001): Cultivation technology for plantation of masson pine. China Agriculture Publishing House (Ed 1). Beijing. pp 122-127.

Zhang, Y., Z. C. Zhou, G. Q. Jin and G. F. QIN (2010): Genetic distances of parents of Pinus massoniana and relationship between growth traits and heterosis of progeny. Journal of Nanjing Forestry University 34: 10-16.

Zhou, L., S. Q. Liu, Y. X. Zhu, Z. Y. Huang and Z. P. ShaO (2008): Relationship between growth traits and growth stress of masson pine. Scientia Silvae Sinicae 44: 102-101.

Zhou, Z. C., G. R. Li, G. L. Huang, B. X. Chen and Y. K. LIN (2000): Genetic control of wood chemical compositions and its implication for wood breeding of masson pine. Scientia Silvae Sinicae 36: 110-117.

\title{
Mendelian segregation in eight microsatellite loci from hand- and open-pollinated progenies of Araucaria angustifolia (Bert.) 0. Kuntze (Araucariaceae)
}

\author{
By M. A. Danner ${ }^{1), *}$, J. Z. Ribeiro ${ }^{1)}$, F. Zanette ${ }^{1)}$, J. V. M. Bittencourt ${ }^{2)}$ and A. M. Sebbenn ${ }^{3}$
}

(Received $11^{\text {th }}$ December 2012)

\begin{abstract}
In order to use molecular markers in population genetics studies, it is important to confirm that the molecular markers used present a Mendelian segregation. The aim of this paper was to investigate the genetic segregation of eight microsatellite loci of Araucaria angus tifolia (Bert.) O. Kuntze (Araucariaceae). The study was carried out comparing genetic segregation in hand- and open-pollinated progenies of maternal dioecious and monoecious trees. The Mendelian segregation was confirmed for all eight loci studied (Ag20, Ag23, Ag45, Aang01, Aang14, Aang28, As90 and CRCAc1), as no deviation from the expected segregation hypothesis was

1) Universidade Federal do Paraná, Centro de Ciências Agrárias, Curitiba, PR, Brazil, CP 19061, 81531-990.

2) Universidade Tecnológica Federal do Paraná, Ponta Grossa, PR, Brazil, 84016-210.

3) Instituto Florestal de São Paulo, São Paulo, SP, Brazil, CP 1322, 01059-970.

*) Corresponding author: Moeses Andrigo Danner. Empresa de Pesquisa Agropecuária e Extensão Rural de Santa Catarina, São Lourenço do Oeste, Santa Catarina, 89990-000, Brazil. E-Mail: moesesandrigo@yahoo.com.br
\end{abstract}

detected in the studied progenies. Therefore, these eight loci can be used for further population genetics studies of $A$. angustifolia.

Key words: Brazilian pine, artificial pollination, open pollination, monoecy.

\section{Introduction}

Araucaria (Araucaria angustifolia (Bert.) O. Kuntze) (Araucariaceae) is a dioecious, wind pollinated conifer that is rarely monoecious. The species is endemic to the South and Southeast of Brazil, with small fragments of the species occurring in Argentina and Paraguay (HUECK, 1972). The species is currently considered endangered (MMA, 2008) since its population area in Brazil has been reduced to less than $3 \%$ of the 18.2 million hectares recorded in the late nineteenth century (HUECK, 1972; GUERRA et al., 2002). The species is ecologically important because adult trees create shady conditions under the canopy which are necessary for the regeneration of other native species (BARBOSA et al., 2009). Moreover, the Araucaria seeds are a crucial source of food for animals that inhabit Araucaria forests 
as the seeds provide an excellent source of energy, particularly in winter (VIDOLIN et al., 2011). Humans also consume the seeds and the harvesting and selling of seeds is a significant source of annual income for hundreds of low-income families in Southern Brazil (SILVA and REIS, 2009).

Knowledge of the mating system, gene flow, genetic structure and diversity of a species are fundamental in developing strategies for the conservation and breeding of tree species (SEBBENN, 2006). Such information can be efficiently assessed based on data from molecular markers, such as SSR (simple sequence repeat), also known as microsatellites. However, for molecular markers to be used in population genetics studies, it is important to confirm that the loci have Mendelian segregation in order to avoid bias in the estimates of genetic parameters (HATTEMER and GILLET, 1989).

Segregation is how a pair of alleles is separated during meiosis in gametes and how alleles are distributed in a given population. To check for deviations in segregation, loci from hand-pollinated progenies of known parental genotypes (full-sib progeny) and open-pollinated progenies from heterozygous maternal genotypes (progeny which may contain mixtures full-sibs, halfsibs, and selfing) can be compared.

The objective of this study was to investigate the segregation of eight microsatellite loci of $A$. angustifolia in order to determine their suitability for future studies of the mating system, gene flow, genetic structure and diversity of the species. The study was conducted comparing the segregation of hand- and open-pollinated progenies from dioecious and monoecious maternal trees.

\section{Material and Methods}

\section{Sampling}

To obtain the $A$. angustifolia progenies, hybridizations were performed between 2005 and 2006, following the methods described in ANSELMINI and ZANETTE (2012). Hand-pollinations were developed (Table 1), using five sampled female individuals (PF_1, PF_3, PF_Social, PF_Solos and PF_Fazenda, all located in the city of Curitiba, Paraná) and six male individuals as pollen donors (Gua_1 and Gua_2, located in the Parque das Araucárias in Guarapuava, Paraná; PB_1 located in Pato Branco, Paraná; and Lg_Galpão, Lg_Horta and Lg_5, from the Experimental Farm Epagri in Lages, Santa Catarina). Hand-pollination was also performed by inducing selfing of the monoecious individual PF_3 (PF_3 x PF_3). Open-pollinated progenies (with no control over male pollen donors) were obtained from three female dioecious trees (Pinha_400, located in Caçador, Santa Catarina; and PF_Social and PF_Fazenda, located in Curitiba, Paraná) and three monoecious trees (MN_Ara from Aratiba, Rio Grande do Sul; MN_SD, São Domingos, Santa Catarina; and MN_Gua, Guarapuava, Paraná). The mature Araucaria cones resulting from both hand- and open-pollination were harvested and the seeds sown in polythene bags containing soil as substrate. The seedlings of each progeny were labeled separately and were grown outdoors in the Agricultural Sciences complex at the Universidade Federal do Paraná (UFPR), in Curitiba, Paraná.

Needles were collected from the sampled parents as well as 18 offspring (2-3 years old) resulting from the each of the hand-and open-pollination, totaling 321 samples. After collection, the needles were placed in individual plastic bags containing silica gel to remove moisture

Table 1. - Method of pollination, identification of and the spatial distance between Araucaria angustifolia parents sampled for the analysis.

\begin{tabular}{|c|c|c|c|}
\hline Progeny & Pemale parent & Male parent & $\begin{array}{c}\text { Spatial distance } \\
\text { between parents }(\mathrm{km})\end{array}$ \\
\hline Hand-pollination & $\mathrm{PF} 3$ & PB 1 & 348.3 \\
\hline Hand-pollination & $\mathrm{PF} 3$ & Gua 1 & 223.3 \\
\hline Hand-pollination & PF_3 & Ig_Galpão & 284.2 \\
\hline Hand-pollination & PF Social & $\mathrm{PB} 1$ & 350.1 \\
\hline Hand-pollination & Pli_Solos & PB_1 & 348.4 \\
\hline Hand-pollination & $\mathrm{Pl}$ _Solos & l.g_Horta & 284.5 \\
\hline Hand-pollination & PF Fazenda & $\operatorname{Lg} 5$ & 291.8 \\
\hline Hand-pollination & PF Fazenda & Gua 2 & 235.3 \\
\hline Hand-pollination & PF_1 & Ig_Horla & 284.4 \\
\hline Hand-pollination & $P l^{2} 3$ & 1.g_Horta & 284.3 \\
\hline Hand-pollination & PF_3 & PF_3 & - \\
\hline Open-pollination & Pinha_400 & - & - \\
\hline Open-pollination & PF_Fayenda & - & - \\
\hline Opcn-pollination & $\mathrm{PF}$ _Social & - & - \\
\hline Open-pollination & $\mathrm{MN}$ N $\mathrm{Nra}$ & - & - \\
\hline Open-pollination & MN_SD & - & - \\
\hline Open-pollination & MN_Gua & - & - \\
\hline
\end{tabular}

PF_3, MN_Ara, MN_SD and MN_Gua are monoecious A. angustifolia trees. 
and prevent degradation of DNA. Samples were shipped to the Laboratory of Genetics of Microorganisms (LabGeM), UFPR, and were subsequently lyophilized for 72 hours and stored at $-40^{\circ} \mathrm{C}$ until DNA isolation.

\section{Microsatellite analysis}

DNA isolation from the A. angustifolia needles was undertaken using the protocol described in FERREIRA and GRATTAPAGLIA (1996, p.127), with minor modifications. Quantification was performed with DNA from each sample using the NanoDrop spectrophotometer ${ }^{\circledR} 2000$ (Thermo Scientific). After quantification, each sample was diluted with autoclaved milli-Q water to a final concentration of $10 \mathrm{ng} / \mathrm{uL}$. This study used eight microsatellite loci developed for the Araucaria species which were chosen based on a reported absence of linkage disequilibrium in the original analyses (ScOTT et al., 2003; SALGueIRo et al., 2005; SchmidT et al., 2007). However, no published study exists that describes the inheritance of these loci. To verify the annealing temperature $\left(\mathrm{Ta}^{\circ} \mathrm{C}\right)$ most suitable for each of the eight loci, we tested 10 DNA samples for each loci using PCR (Polymerase Chain Reaction) and temperature gradients in the Maxygene ${ }^{\circledR}$ thermocycler (Axygen). The PCR product was visualized by electrophoresis on $1.5 \%$ agarose gel, applying $5 \mu \mathrm{L}$ of the PCR product and $2 \mu \mathrm{L}$ of the GelRed ${ }^{\circledR}$ (Biotium) dye. Electrophoresis was performed with a current of 3 volts/cm for $120 \mathrm{~min}$ and the result was visualized using transillumination with ultraviolet light.

For PCR amplification of all samples we used a reaction solution with a final volume of $10 \mu \mathrm{L}$, containing $5 \mu \mathrm{L}$ of Qiagen Multiplex PCR Master Mix 2X, $1 \mu \mathrm{L}$ of 10X primer mix ( $2 \mathrm{mM}$ each primer), $2 \mu \mathrm{L}$ genomic $\mathrm{DNA}$ (10 ng l-1), $1 \mu \mathrm{L}$ of $5 \mathrm{X}$ Q-Solution, and $1 \mu \mathrm{L}$ of Milli-Q water. The PCR program was used in the thermocycler with an initial step of denaturation of DNA and Taq DNA polymerase activation at $95^{\circ} \mathrm{C}$ for $15 \mathrm{~min}$, followed by 35 cycles of amplification in three stages $\left(94^{\circ} \mathrm{C}\right.$ for $30 \mathrm{sec}$ ), annealing temperature (Table 2) for $90 \mathrm{sec}$, and $72^{\circ} \mathrm{C}$ for 60 seconds), and a final extension at $72^{\circ} \mathrm{C}$ for
10 minutes. After amplification we added $10 \mu \mathrm{L}$ of Milli$\mathrm{Q}$ water to each sample, which were subsequently kept refrigerated at $4{ }^{\circ} \mathrm{C}$ until genotyping.

For genotyping of each sample, we used a solution of $10 \mu \mathrm{L}$ containing $1 \mu \mathrm{L}$ of the amplified fragment solution, $0.125 \mu \mathrm{L}$ of GS $500 \mathrm{ROX}^{\circledR}$ standard or $0.5 \mu \mathrm{L}$ of GS $600 \mathrm{LIZ}^{\circledR}$, and the remaining volume was made up with formamide $\mathrm{Hi}-\mathrm{Di}^{\circledR}$. Polymorphism was detected by labeling primers and PCR biplex or triplex combinations with fluorescent dyes (Table 2), followed by detection of the fragments by capillary electrophoresis in $3500 \mathrm{xL}$ ABI automated sequencer Genetic Analyzer (Applied Biosystems). The size of the fragments (alleles) were determined by interpretation of the electropherogram peaks generated using the GeneMapper v.4.1 software (Applied Biosystems). The values referring to the sizes of the alleles were exported to spreadsheets for statistical analysis.

\section{Data analysis}

To study the segregation of microsatellite loci in A. angustifolia, we adopted the method described by Gillet and HatTEMER (1989). We compared the maternal and paternal genotypes (in cases of controlled parentage) with the segregation of the hand- and openpollinated progeny. The test requires that the following conditions must be met. Firstly, all progeny of a tree $A_{i} A_{i}$ must possess the $A_{i}$ allele from the mother tree. Secondly, in cases of heterozygous parent trees (e.g. $A_{i} A_{i}, \mathrm{i} \neq \mathrm{j}$ ): a) among offspring, each individual must possess an allele of the maternal tree, $A_{i}$ and $A_{j}$; b) the number of heterozygous progeny $A_{i} A_{j}\left(n_{i j}\right)$ must equal the sum of homozygous progeny $A_{i} A_{i}\left(n_{i i}\right)$ and $A_{j} A_{j}\left(n_{j j}\right)$, or $n_{i j}=n_{i i}+n_{i j}$; and c) the number of heterozygous progeny $A_{i} A_{k}\left(n_{i k}\right)$ must equal the number of heterozygous progeny $A_{j} A_{k}\left(n_{j k}\right)$, or $n_{i k}=n_{j k}$, where $k \neq \mathrm{i}, \mathrm{j}$. The phenotypes observed in each heterozygous offspring were compared with the expected segregation by event of $1: 1$ or $1: 2: 1$, by means of a maximum likelihood G-test using the following formula:

Table 2. - Primers labeled with fluorescence without combination or in biplex or triplex combination for genetic analysis of $A$. angustifolia.

\begin{tabular}{lccc}
\hline \multicolumn{1}{c}{ Combination } & Fluoresence & Detected fragment (pb) & Annealing temperature \\
\hline Ag20 & FAM (bluc) & $240-258$ & $59.3^{\circ} \mathrm{C}$ \\
Triplex & FAM (bluc) & $154-168$ & $57.8^{\circ} \mathrm{C}$ \\
Ag45 & FAM (blue) & $203-225$ & $57.8^{\circ} \mathrm{C}$ \\
CRCAc) & Fl:D (yellow) & $160-180$ & $57.8^{\circ} \mathrm{C}$ \\
As90 & & \\
Biplex & HEX (green) & $200-260$ & $56^{\circ} \mathrm{C}$ \\
Aang01 & FAM (bluc) & $150-190$ & $56^{\circ} \mathrm{C}$ \\
Aang14 & & & $55^{\circ} \mathrm{C}$ \\
Biplex & HEX (green) & $130-170$ & $55^{\circ} \mathrm{C}$ \\
Aang28 & FAM (blue) & $245-259$ & $\mathrm{G}$ \\
Ag23 & & & \\
\hline
\end{tabular}

$500 \mathrm{ROX}^{\circledR}$ GS was used to size samples with the loci Ag20, Ag45, As90 and CRCAc1 and GS 600 LIZ $^{\circledR}$ for samples with loci Ag23, Aang01, Aang14 and Aang28. 


$$
G=2\left[n_{i} \ln \left(\frac{n_{i}}{E(n)}\right)+n_{j} \ln \left(\frac{n_{j}}{E(n)}\right)\right]
$$

where, $n_{i}$ and $n_{j}$ are the observed number of genotypes containing alleles $A_{i}$ and $A_{j}$, respectively, $E(n)$ is the expected number of genotypes for the allele $A_{i}$ and $A_{j}$, where $E(n)=0.5\left(n_{i}+n_{j}\right)$, ln is the natural logarithm. The $G$-test determines if the deviation between the observed and expected segregation is statistically significant or if deviations may be explained by chance. We also applied the Bonferroni correction for multiple comparisons $(95 \%, \alpha=0.05)$ to avoid false positives. The frequency of alleles at all loci were calculated and classified into three categories: (a) high frequency $(p>0.25)$; (b) inter- mediate frequency $(0.05<p \leq 0.25)$; and (c) rare frequency $(p \leq 0.05)$. The alleles unique to each progeny type were also identified

\section{Results and Discussion}

Of the 321 genotyped samples, only seven $(2.2 \%)$ did not demonstrate sufficient quality for unambiguous genotype detection. This result does not significantly interfere with the segregation analysis.

Among open-pollinated progenies, all loci had private alleles except for CRCAc1. Among hand-pollinated progenies, only two private alleles were found: one allele in the Aang14 locus and one in the Aang28. All loci also showed one or two alleles with high frequency $(p>0.25)$,

Table 3. - Allele frequencies and private alleles of eight microsatellite loci in hand-pollinated progenies $(n=192)$ and open-pollinated progenies $(n=107)$ of Araucaria angustifolia.

\begin{tabular}{|c|c|c|c|c|c|c|c|}
\hline Locus & Allele & Hand-pollination & Open-pollination & I Locus & Allele & Hand-pollination & Open-pollination \\
\hline \multirow[t]{7}{*}{ Ag20 } & 239 & 0.05 & 0.07 & \multirow[t]{7}{*}{$\operatorname{Ag} 23$} & 247 & 0.07 & 0.09 \\
\hline & 241 & 0.08 & 0.06 & & 249 & 0.55 & 0.44 \\
\hline & 243 & 0.71 & 0.67 & & 251 & 0.04 & 0.06 \\
\hline & 245 & 0.00 & 0.01 & & 253 & 0.07 & 0.07 \\
\hline & 249 & 0.16 & 0.16 & & 255 & 0.27 & 0.28 \\
\hline & 253 & 0.00 & 0.03 & & 257 & 0.00 & 0.06 \\
\hline & Private & 0 & 2 & & Private & 0 & 1 \\
\hline \multirow[t]{4}{*}{ Ag45 } & 151 & 0.00 & 0.04 & \multirow[t]{3}{*}{$\mathrm{CRCAcl}$} & 199 & 0.10 & 0.14 \\
\hline & 163 & 0.19 & 0.17 & & 201 & 0.79 & 0.74 \\
\hline & 165 & 0.81 & 0.79 & & 203 & 0.11 & 0.12 \\
\hline & Private & 0 & 1 & & Private & 0 & 0 \\
\hline \multirow[t]{18}{*}{ Aang01 } & 200 & 0.00 & 0.02 & \multirow[t]{17}{*}{ Aang 14} & 148 & 0.00 & 0.02 \\
\hline & 202 & 0.00 & 0.07 & & 150 & 0.02 & 0.04 \\
\hline & 204 & 0.00 & 0.02 & & 152 & 0.09 & 0.15 \\
\hline & 206 & 0.00 & 0.02 & & 154 & 0.00 & 0.01 \\
\hline & 208 & 0.32 & 0.07 & & 156 & 0.00 & 0.01 \\
\hline & 210 & 0.02 & 0.01 & & 160 & 0.00 & 0.01 \\
\hline & 212 & 0.04 & 0.10 & & 162 & 0.04 & 0.07 \\
\hline & 214 & 0.00 & 0.20 & & 164 & 0.29 & 0.26 \\
\hline & 216 & 0.17 & 0.10 & & 168 & 0.15 & 0.05 \\
\hline & 218 & 0.23 & 0.11 & & 170 & 0.26 & 0.25 \\
\hline & 220 & 0.02 & 0.05 & & 172 & 0.00 & 0.08 \\
\hline & 222 & 0.06 & 0.04 & & 174 & 0.06 & 0.02 \\
\hline & 224 & 0.11 & 0.09 & & 176 & 0.00 & 0.01 \\
\hline & 226 & 0.02 & 0.01 & & 180 & 0.00 & 0.01 \\
\hline & 230 & 0.00 & 0.07 & & 182 & 0.02 & 0.02 \\
\hline & 232 & 0.00 & 0.01 & & 184 & 0.06 & 0.00 \\
\hline & 244 & 0.00 & 0.01 & & 188 & 0.00 & 0.01 \\
\hline & Private & 0 & 8 & \multirow{13}{*}{$A \mathrm{~s} 90$} & Private & 1 & 8 \\
\hline \multirow[t]{13}{*}{ Aang28 } & 134 & .00 & 0.02 & & 173 & 0.05 & 0.08 \\
\hline & 136 & 0.06 & 0.00 & & 175 & 0.31 & 0.32 \\
\hline & 144 & 0.00 & 0.01 & & 177 & 0.44 & 0.22 \\
\hline & 150 & 0.13 & 0.18 & & 179 & 0.06 & 0.09 \\
\hline & 152 & 0.12 & 0.22 & & 181 & 0.14 & 0.22 \\
\hline & 154 & 0.21 & 0.07 & & 183 & 0.00 & 0.06 \\
\hline & 156 & 0.02 & 0.03 & & 189 & 0.00 & 0.01 \\
\hline & 158 & 0.04 & 0.02 & & - & - & - \\
\hline & 160 & 0.00 & 0.01 & & - & - & - \\
\hline & 162 & 0.07 & 0.09 & & - & - & - \\
\hline & 166 & 0.26 & 0.12 & & - & - & - \\
\hline & 168 & 0.10 & 0.23 & & - & - & - \\
\hline & Private & 1 & 3 & & Private & 0 & 2 \\
\hline
\end{tabular}


except for the open-pollinated progenies with locus Aang01, for which there were eight alleles of intermediate frequency $(0.05<p \leq 0.25)$ and nine rare alleles $(p>0.05)$. In some loci, no rare alleles were found, including the hand-pollinated progenies with loci Ag45 and CRCAc1, and open-pollinated progenies with loci Ag23 and CRCAc1 (Table 3). The loss of low frequency alleles in fragmented $A$. angustifolia populations at a rate higher than continuous, well preserved forest (e.g. Mangueirinha, Paraná) was reported in BitTEncourT and SEBBEnN (2009). Therefore, the presence of private and low frequency alleles detected in the current study demonstrates the potential of open-pollinated progenies for ex situ genetic conservation and breeding programs. However, it should be noted that these progenies are the maternal sibs of only six trees (three female and three monoecious), located in three different Brazilian states. Thus, the differentiation of alleles is expected due to the effects of long distances (WRIGHT, 1943). Although the microsatellite markers used in this study are neutral,

Table 4. - Mendelian inheritance in eight microsatellite loci in hand-pollinated progenies of Araucaria angustifolia.

\begin{tabular}{|c|c|c|c|c|c|c|c|c|c|c|c|c|c|c|}
\hline $\begin{array}{l}\text { Locusillybrid } \\
\text { Agr20 }\end{array}$ & Gicnolype & $n$ & $n_{i i}: n_{i:}+n_{i i}$ & $n_{i \hbar}: n_{j i k}$ & $n_{i !}: n_{1 ! i}$ & $G$ & $D f$ & $\begin{array}{l}\text { Loeusitlybrid } \\
\text { Agt5 }\end{array}$ & Genotype & $n$ & $n_{i j}: n_{i i}+n_{y j}$ & $n_{i, k}: n_{j, k}$ & $n_{i l}: n_{i i}$ & $G$ \\
\hline P]: $3 x p+3 \quad 1$ & $243 / 24 \% \times 243 / 249$ & 18 & $10: 8$ & $0: 0$ & $0: 0$ & 2.32 & 2 & $\mathrm{PF}=3 \times \mathrm{PM}^{\mathrm{B}} \mathrm{I}$ & $163 / 165 \times 165 / 165$ & 18 & $7: 22$ & $0: 0$ & $0: 0$ & 0.90 \\
\hline PF 3xGua 1 & $243 / 249 \times 230 / 243$ & 17 & $7: 6$ & $2: 2$ & $0: 0$ & 5.09 & 3 & PF 3xGua 1 & $163 / 165 \times 1631165$ & 17 & $4: 13$ & $0: 0$ & $0: 0$ & 5.72 \\
\hline Pr_3XLE_Gialpão & $2431249 \times 239 / 243$ & 18 & 5.5 & $5: 3$ & $0 \cdot 11$ & 0.73 & 3 & PF_3xLg_Ciápầ & 163165163165 & 18 & $4: 14$ & $0: 0$ & 0.0 & 5.66 \\
\hline P]:_Social xPH_1 & $239 / 241 \times 243 / 249$ & 17 & $(1 ; 0)$ & $6: 5$ & $2: 4$ & 2.26 & 3 & $\mathrm{PF}$ _SocialxPE_] & $163 / 165 \times 1651165$ & 17 & $7: 10$ & $0: 0$ & $0 ; 6$ & 0.53 \\
\hline PF_SolusxPB_1 & $241 / 24.7 \times 243 / 249$ & 17 & $4: 3$ & $0 \div 0$ & $4: 6$ & 1.08 & 3 & 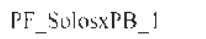 & $165 / 165 \times 165 / 165$ & 17 & $0: 17$ & $0: 0$ & 0,0 & - \\
\hline Pf Sulosxlg lorta & $241 / 243 \times 243 / 243$ & 16 & $5: 11$ & $0: 0$ & $0:(1)$ & 2.31 & ] & P'F sulosxl thorla & $165 / 165 \times 165: 165$ & 16 & $0: 16$ & $0: 0$ & $0: 4$ & - \\
\hline Pl:_lazendaxLg_5 & $243 / 243 \times 241 / 243$ & 18 & $0: 10$ & $8: 0$ & $0: 0$ & 0.22 & 1 & Pl:_lazcndarLg_5 & $165 / 165 \times 163 / 165$ & 18 & $0: 10$ & $8: 0$ & $0: 0$ & 0.22 \\
\hline P]: Faxendaxciua 2 & $24.324 .3 \times 243243$ & 17 & $0: 17$ & $0: 0$ & $0: 0$ & - & - & PF FaxendaxG raa 2 & $165 / 65 \times 165 i 165$ & 17 & $0: 17$ & $0: 0$ & $0: 0$ & - \\
\hline PF $\mid x]$ e I Inorta & $24,3 / 24,3 \times 24,3 / 24,3$ & 18 & $0: 18$ & $0: 0$ & $0: 0$ & - & - & $\mathrm{PF}|\mathrm{x}|_{-2 y}$ florta & $16516,5 \times 165: 165$ & 18 & $(0: 18$ & $0: 0$ & $0 ; 0$ & - \\
\hline PJ:_3xLE_Horla & $243 \cdot 249: 2437243$ & 18 & 612: & 0:0 & $0: 11$ & 2.04 & 1 & 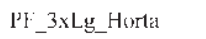 & $163: 165 \times 1651165$ & 18 & $7: 11$ & $0: 10$ & $0: 0$ & 10.90 \\
\hline PF $3 x P F$ & $243 / 249 \times 243 / 249$ & 18 & $6: 12$ & $0: 0$ & $0: 0$ & 2.04 & 2 & $\mathrm{PF} 3 \times \mathrm{PF}^{\mathrm{P}} 3$ & $163: 165 \times 163 / 165$ & 18 & $9: 9$ & $0: 0$ & $0: 4$ & 0.00 \\
\hline $\operatorname{sg} 23$ & & & & & & & & Aang01 & & & & & & \\
\hline$\left.P F=\left.3 x\right|^{\prime} B_{-}\right]$ & $249 / 255 \times 249 / 253$ & 18 & $5: 4$ & $0: 0$ & $5: 4$ & 0.22 & 3 & $\mathrm{I}^{\prime} \mathrm{H}_{-}^{-} 3 \mathrm{P}^{\mathrm{B}} \mathrm{B}-\mathrm{I}$ & $208 / 218 \times 2088216$ & 18 & 6.6 & $0: 0$ & $3: 3$ & 2.04 \\
\hline P]: 3xGua 1 & $249 / 255 \times 240 / 249$ & 17 & $7: 10$ & $0: 0$ & $0: 0$ & 0.5 .3 & ] & PF $3 \times$ Xikat 1 & $208 / 218 \times 208 / 222$ & 17 & $5: 4$ & $0: 0$ & $4: 4$ & 0.17 \\
\hline РГ 3x丁g Chlpăo & $249 / 255 \times 249 / 251$ & 18 & $4: 5$ & $0: 0$ & $4: 5$ & 0.22 & 3 & PF 3xI g Gralpâo & $208 / 218 \times 212 / 212$ & 18 & $0: 0$ & $10: 8$ & $0: 0$ & 0.22 \\
\hline Pr_Sociul XPB_1 & $249 / 255 \times 240 / 253$ & 17 & $5: 4$ & $0: 0$ & $4: 4$ & 0.17 & 3 & PF_Socialxpes_l & $216220 \times 208: 216$ & 17 & $5: 4$ & $4: 4$ & 000 & 0.17 \\
\hline Pl:_SoloskPB3_l & $247 / 255 \times 249 / 253$ & 17 & 0.5 & $5: 4$ & $5: 3$ & 0.68 & 3 & $\left.\mathrm{PF}_{-} \mathrm{S}_{\operatorname{sil} l o s x} \mathrm{~PB} \mathrm{~B}_{3}\right]$ & $218: 222 \times 2088: 216$ & 17 & $0: 0$ & $5: 4$ & $4: 7$ & 0.17 \\
\hline PF_SolusxLE_Herta & $247 / 255 \times 240 / 249$ & 16 & $0: 0$ & $8: 8$ & $0: 0$ & 0.00 & 1 & PF_SulasxLe_Horta & $218222 \times 216224$ & 16 & $0: 0$ & $4: 4$ & $4: 4$ & 0.00 \\
\hline Pf Faxendaxl 135 & $249 / 255 \times 247249$ & 18 & $5: 4$ & 5.4 & $0: 0$ & 0.32 & 3 & Pr Furendaxis 5 & $208224 \times 208210$ & 18 & $5: 0$ & $0: 0$ & $4: 5$ & 0.22 \\
\hline Pl:_razendaxGua_z & $249 / 255 \times 249 / 251$ & 17 & $5: 4$ & 0.0 & $4: 4$ & 0.17 & 3 & Pl__accndaxGua_2 & $208224 \times 218 / 218$ & 17 & $0: 0$ & $9: 8$ & 0.0 & 0.06 \\
\hline PJ:_IXLE_Horla & $249 / 255 \times 24 \% / 249$ & 18 & $10: 8$ & $0: 0$ & $0 ; 1)$ & 0.22 & 1 & 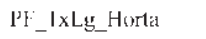 & $216 / 226 \times 216224$ & 18 & $4: 5$ & $0 ; 1)$ & $4: 5$ & 0.22 \\
\hline PF_3xLE__Jorta & $249 / 255 \times 249 / 249$ & 18 & $10: 8$ & $0: 0$ & $0: 0$ & 0.22 & 1 & 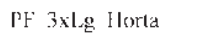 & $208: 218 \times 216: 224$ & 18 & $0: 0$ & $4: 6$ & $4: 4$ & 0.63 \\
\hline $\mathrm{Pl}: 3 \times \mathrm{PF} \quad 3$ & $249 / 255 \times 240 / 255$ & 18 & $8: 4$ & $0: 0$ & $0: 0$ & 0.22 & 2 & $\mathrm{PF} 3 \mathrm{xPF} 3$ & $208218 \times 208 / 218$ & 18 & $5: 13$ & $0: 0$ & $0: 6$ & 3.68 \\
\hline Aang $\mid 4$ & & & & & & & & As 90 & & & & & & \\
\hline 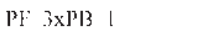 & $164: 170 \times 168 / 184$ & 18 & $0: 0$ & $5: 5$ & $4: 7$ & 0.22 & 3 & $\mathrm{Pl}: 3 \times \mathrm{P}] 3 \quad 1$ & $175 / 177 \times 177 / 177$ & 18 & $12: 6$ & $0:[5$ & $0: 0$ & 2.04 \\
\hline PF 3xGua 1 & $164: 170 \times 152 / 64$ & 17 & $4: 5$ & $4: 4$ & $0: 0$ & 0.17 & 3 & PF 3xGrua 1 & $175: 177 \times 175: 181$ & 17 & $3: 3$ & $0: 0$ & $6: 5$ & 1.58 \\
\hline Pr_3xLg_Galpão & $164 / 170 \times 164 / 182$ & 18 & $7: 2$ & $0: 0$ & $4: 5$ & 3.05 & 3 & P'E_3xLg_Galpão & $175 / 177 \times 177 / 177$ & 18 & $8: 10$ & $0: 0$ & $0: 0$ & 0.22 \\
\hline PI:Social $\times P B$ _I & $164 / 170 \times 1688184$ & 17 & $0: 0$ & $4: 6$ & $3: 4$ & 1.08 & 3 & $\mathrm{Pl}_{-}$ScocialxPl3_l & $173: 175 \times 177 / 177$ & 17 & $0 ; 0$ & $8: 9$ & $0: 0$ & 0,06 \\
\hline PF_SolusxPB_1 & $152 / 174 \times 168: 184$ & 17 & $0: 0$ & $5: 3$ & $6: 3$ & 1.58 & 3 & PF_SolosxPB_1 & $179: 181 \times 177 / 177$ & 17 & $0: 0$ & $9: 0$ & $0: 0$ & 0.66 \\
\hline I'F' SolessxL_y' Florlit & $152: 174 \times 164: 168$ & 16 & $0: 0$ & $5: 4$ & $3: 4$ & $0.5]$ & 3 & PF SolosxI g Hental & $179 / 181 \times 175 / 175$ & 16 & (nos & $7: 9$ & $0: 0$ & 0.25 \\
\hline PF FayendaxLs 5 & $162: 170 \times 164: 170$ & 18 & $3: 9$ & $4: 6$ & $0: 0$ & 1.13 & 3 & PF Farendaxi g 5 & $177 / 181 \times 177 / 181$ & 18 & $7: 11$ & $0: 0$ & $0: 0$ & 0.99 \\
\hline PF_Farendiarojua_2 & $162170 \times 150 \% 170$ & 17 & $4: 9$ & $5: 4$ & $0: 0$ & 0.17 & 3 & P'F_fax.tondaxtiwa_2 & $177: 181 \times 177 / 179$ & 17 & $4: 4$ & 0:0 & $4: 5$ & 0.17 \\
\hline 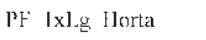 & $152174 \times 164168$ & 18 & $0: 0$ & $5: 5$ & 3.5 & 0.73 & .3 & PF $1 \times$ Lg l Iortal & $173: 177 \times 175: 175$ & 18 & tho & $10: 8$ & $0: 0$ & 0.22 \\
\hline PF $3 x-g$ Horta & $164: 170 \times 164 / 68$ & 18 & $4: 5$ & $0: 0$ & 4.5 & 0.22 & 3 & $\mathrm{PF} 3 \times[\mathrm{g}$ Horta & $175: 177 \times 175 / 175$ & 18 & $11: 7$ & $0: 0$ & $0 ; 0$ & 0,90 \\
\hline 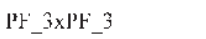 & $164 / 170 \times 164 / 170$ & 18 & $8: 5$ & $0: 0$ & 0.0 & 0.22 & 2 & $\mathrm{PH}_{-} 3 \times \mathrm{P}^{\mathrm{P}} \mathrm{F}_{-} 3$ & $175 / 177 \times 175 / 177$ & 18 & $7: 11$ & $0: 0$ & $0: 0$ & 0.90 \\
\hline $\operatorname{Aang} 28$ & & & & & & & & CRCAel & & & & & & \\
\hline $\mathrm{PF} 3 \mathrm{xPB} 1$ & $154: 166 \times 136: 168$ & 18 & $0: 0$ & $4: 4$ & $5: 5$ & 0.22 & 3 & $\mathrm{PF} 3 \times \mathrm{PB} 1$ & $201 / 201 \times 159 / 201$ & 18 & 0.5 & $9: 0$ & $0: 0$ & 0.60 \\
\hline Pr_3xGua_l & $154166 \times 156 / 162$ & 17 & $0: 0$ & $5: 4$ & $4: 7$ & 0.17 & 3 & 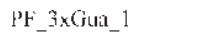 & $201 / 201 \times 201 / 201$ & 17 & $0: 17$ & $0: 0$ & $0: 0$ & - \\
\hline PI:_ixLg_Galpñth & $154166 \times 152 / 68$ & 18 & $0: 0$ & $4: 5$ & $5: 4$ & 0.22 & 3 & $\mathrm{Pl}_{-} 3 \times \mathrm{Lg}_{-} \mathrm{C}$ (ialpano & $201 / 201 \times 201 / 203$ & 18 & $0: 11$ & $0: 0$ & $7: 0$ & 0.90 \\
\hline PF_SocialxPB_I & $150 / 152 \times 136 / 168$ & 17 & $0: 0$ & $4: 4$ & $4: 5$ & 0.17 & 3 & PF_SckialxPB_1 & $199 / 201 \times 159: 201$ & 17 & $7: 10$ & (0:0) & $0: 0$ & 0.93 \\
\hline $\mathrm{P}^{\mathrm{P}}$ Sols $\mathrm{XP} B$ & $15215 \times \times 136 \% 168$ & 17 & $0: 0$ & $4: 4$ & $5: 4$ & 0.17 & 3 & PF SolowXPH 1 & $201 / 201 \times 190 / 201$ & 17 & 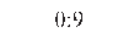 & $x: 0$ & $0: 0$ & 0.06 \\
\hline PF Solosx D Elortit & $152158 \times 150 / 66$ & 16 & $0: 0$ & $4: 4$ & $4: 4$ & 0.00 & 3 & PF SolosxI g Horta & $201 / 201 \times 201 / 203$ & 16 & 0,9 & $0: 0$ & $7: 0$ & 0.25 \\
\hline P'F_Fuxendix Lg_5 & $154 / 162 \times 150 / 166$ & 18 & $0: 0$ & $5: 4$ & $5: 4$ & 0.22 & 3 & P'F_faxtondaxLL_5 & $201: 201 \times 201 / 203$ & 18 & $0: 7$ & $0: 0$ & $11: 0$ & 0.90 \\
\hline PF lidrendaxGiua 2 & $154 / 162 \times 150 / 152$ & 17 & $0: 0$ & $4: 4$ & $4: 5$ & 10.17 & 3 & Pli diarendaxciua 2 & $201: 201 \times 201 / 201$ & 17 & $0: 17$ & $0:[1$ & $0: 0$ & - \\
\hline PF $\mid x]_{\text {go }}$ Horta & $154: 166 \times 150 / 166$ & 18 & $5: 4$ & $4: .5$ & $0: 0$ & 0.22 & 3 & PF 1x[.g Horta & $199 / 201 \times 201203$ & 18 & $4: 5$ & $0: 0$ & $4: 5$ & 0.22 \\
\hline PF_3xL的_Horta & $154 / 166 \times 150 / 166$ & 18 & $4: 5$ & $4: 5$ & $0: 0$ & 0.22 & $\hat{3}$ & PF_3x[e_ITorta & $201 / 201 \times 201 / 203$ & 18 & $0: 11$ & $0: 0$ & $7: 0$ & 0.90 \\
\hline $\mathrm{PJ}: 3 \times \mathrm{xPl}: 3$ & $154 / 166 \times 154 / 166$ & 18 & $9: 9$ & $0 ; 0$ & $0: 0$ & 0.00 & 2 & $\mathrm{Pl}: 3 \mathrm{KPl}^{\circ} 3$ & $201 / 201 \times 201 / 201$ & 18 & $0: 18$ & $0: 0$ & $0: 0$ & - \\
\hline
\end{tabular}

$n$ is the sample size. $G$ is the value of the maximum likelihood $G$-test for the hypotheses $n_{i f}=n_{i i}+n_{i j}$ and $n_{i k}=n_{j k} . d f$ are the degrees of freedom. In progenies with $2 d f$, the segregation tested hypothesis was 1:2:1 and in other progenies the tested hypothesis was 1:1. 
other alleles that are part of gene regions affected by selection may have been inherited by the progeny. These can have a significant impact on traits for adaptation and breeding of Araucaria. According to BERGMANN et al. (1990), rare alleles in a species, but with intermediate frequency in a population may be important for the performance and adaptability of a population in the long term.

Compared to other studies carried out using microsatellite markers for A. angustifolia, our study showed a lower number of alleles for the loci Ag20, Ag23, As90 and CRCAc1, and a greater number of alleles for the loci Aang14 and Aang28. Our results are consistent with most studies for the locus Aang01, which showed the highest number of alleles among all loci (SCHMidT et al., 2007; StEFEnON et al., 2007; SANT'ANNA et al., 2013). However, PATREZE and TSAI (2010) reported that the locus Aang01 had a lower number of alleles than the other five loci used in their study.

No significant deviations from the expected segregation hypothesis (1:1 or 1:2:1) were detected in any of the hand- or open-pollinated progeny sampled (Tables 4 and 5). The highest values of the $G$-test were found for the loci Ag45, among the hand-pollinated progenies PF_3 x Gua_1 $(\mathrm{G}=5.72)$ and PF_3 x Lg_Galpão $(\mathrm{G}=5.66)$.

Allele 136 in the locus Aang28 was found only in the male parent PB_1 sampled for hand-pollination and in its progeny. This demonstrates the ability of hand-pollination strategies to form new genotypes, since in a natural setting no hybridization could be accomplished due to the significant distance between the paternal and maternal trees sampled.
In the case of locus Ag23, maternal trees showed a low diversity of alleles. Of the nine genotyped trees with this locus, seven have the same genotype (249/255), except the dioecious tree PF_Solos (247/255) and monoecious tree MN_Ara (249/249). This low polymorphism may be due to sampling, as other studies have found greater variation in maternal trees for this same locus (BITTENCOURT, 2007; SANT'ANNA et al., 2013). Conversely, for locus Ag23, both genotyped (hand-pollinated) and nongenotyped (open-pollinated) pollen donors were important for increasing the number of different alleles in the progenies.

In locus As90, allele 183 was unique in the open-pollinated progeny of the seed tree MN_SD. Although this locus was developed for Araucaria subulata, from New Caledonia, it has been shown to be transferable and polymorphic for $A$. angustifolia (BITTENCOURT and SEBBENN, 2007).

The locus CRCAc1 also showed a low number of alleles (three). Only the male parents were found to have allele 203, which again demonstrates the importance of hand-pollination in the formation of new genotypes. We noted that of the nine trees used as maternal parents, only three were heterozygous (PF_Social, PF_1 and MN_Gua) for this locus with genotype 199/201, while the others showed homozygosity with 201/201. BITTENCOURT (2007) also reported that most of the genotyped maternal trees of $A$. angustifolia used in that study were homozygous for the locus CRCAc1, with only two trees showing heterozygosity (200/202). ScOTT et al. (2003) developed the locus CRCAc1 for Araucaria cunninghamii and found that the sequences flanking this site are highly preserved and therefore transferable to

Table 5. - Mendelian inheritance in eight microsatellite loci in open-pollinated progenies of Araucaria angustifolia.

\begin{tabular}{|c|c|c|c|c|c|c|c|c|c|c|c|}
\hline $\begin{array}{l}\text { Locus/Seed tree } \\
\text { Ag20 }\end{array}$ & $\begin{array}{l}\text { Seed tree } \\
\text { genolype }\end{array}$ & $n$ & $n_{i j}: n_{i j}-n_{i i}$ & $n_{i,}: n_{i k}$ & $G$ & $\begin{array}{l}\text { Locus/Seed uree } \\
\text { Aang28 }\end{array}$ & $\begin{array}{l}\text { Seed tree } \\
\text { genotype }\end{array}$ & $n$ & $n_{i j}: n_{i i}+n_{i i}$ & $n_{i k}: n_{i k}$ & $G$ \\
\hline PF_Social & $239 / 241$ & 18 & $4: 2$ & $7: 5$ & 0.00 & PE_Faxenda & $154 / 162$ & 17 & $0: 0$ & $11: 6$ & 1.44 \\
\hline$\Lambda g 23$ & & & & & & Pl_Social & $150 / 152$ & 18 & $0: 0$ & $10: 8$ & 0.22 \\
\hline PF_Faremda & $249: 255$ & 17 & $3: 0$ & $9: 5$ & 1.16 & P'inha_400 & $150 / 152$ & 18 & $1: 0$ & $8: 9$ & 0.06 \\
\hline PI Social & $249 / 255$ & 18 & $3: 0$ & $8: 7$ & 0.29 & $\mathrm{MN} \mathrm{SD}$ & $150 / 168$ & 18 & $1: 7$ & $5: 5$ & 0.06 \\
\hline Pinha 400 & $249: 255$ & 18 & $3: 2$ & $6: 7$ & 0.07 & MN Gua & $152: 162$ & 18 & 46 & $6: 2$ & 1.16 \\
\hline MN SD & $249: 255$ & 18 & $5 \% 7$ & $3: 3$ & 0.08 & $A 590$ & & & & & \\
\hline MN_Gua & $249 / 255$ & 18 & $3: 7$ & $5: 3$ & 0.60 & l't'_f'arenda & $177 / 181$ & 17 & $3: 2$ & 7.5 & 1.16 \\
\hline$A g 45$ & & & & & & PF Social & $173 / 175$ & 18 & 7.3 & $4: 4$ & 0.09 \\
\hline Pr_Social & $239 / 241$ & 18 & $8: 10$ & $0: 0$ & 0.22 & Pinha_400 & $175: 177$ & 18 & $3: 2$ & $7: 6$ & 0.60 \\
\hline Aangol & & & & & & $\mathrm{MK} A \mathrm{ra}$ & $175 / 181$ & 18 & $5: 8$ & $3: 2$ & 0.08 \\
\hline $\mathrm{Pl}^{\mathrm{P}}{ }_{-} \mathrm{l}$ azenda & $208 / 224$ & 17 & $0: 0$ & $8: 9$ & 0.06 & $M N \_S D$ & $179 / 183$ & 18 & $3: 4$ & $7: 4$ & 0.07 \\
\hline PJ: Social & 2161220 & 18 & $1: 0$ & $10: 7$ & 0.53 & MN Giua & $175 / 181$ & 18 & $3: 6$ & $5: 4$ & 0.07 \\
\hline Pitha_ 400 & $214: 216$ & 18 & $4: 1$ & $10: 3$ & 4.86 & CRCAcl & & & & & \\
\hline $\mathrm{MN} \mathrm{Ar}_{\Gamma^{\prime}+\mathrm{t}}$ & 202214 & 18 & $1: 6$ & $4: 7$ & 1,49 & PF Social & $199 / 201$ & 18 & 7.3 & 35 & 2.36 \\
\hline MN_Gua & $212 / 230$ & 18 & $2: 5$ & $2: 9$ & 2.31 & MN_Gua & $199 / 201$ & 18 & 7.5 & $5: 1$ & 0.09 \\
\hline \multicolumn{12}{|l|}{ Aang 14} \\
\hline $\mathrm{Pl}^{\mathrm{l}}$ _lazenda & $162 / 170$ & 17 & (0:0) & $8: 9$ & 0.06 & & & & & & \\
\hline Pl' Social & $164: 170$ & 18 & $0: 0$ & $9: 9$ & 0.000 & & & & & & \\
\hline Pinha 400 & $150 / 164$ & 18 & 1,0 & $6: 11$ & 0.90 & & & & & & \\
\hline MN Ara & $152: 172$ & 18 & $4: 3$ & $3: 8$ & 2.66 & & & & & & \\
\hline $\mathrm{MN} \mathrm{SI})$ & 152170 & 18 & $4: 5$ & $2: 7$ & 2.66 & & & & & & \\
\hline MN_iua & $164: 170$ & 18 & $7: 6$ & $4: 1$ & 0.83 & & & & & & \\
\hline
\end{tabular}

$n$ is the sample size. $G$ is the value of the maximum likelihood $G$-test for the hypotheses $n_{i j}=n_{i i}+n_{j j}$ and $n_{i k}=n_{j k}$. 
four species of the genus (A. columnaris, A. heterophylla, A. luxurians and A. bidwillii) and also for Aghatis robusta (Araucariaceae family). According to Scott et al., the low microsatellite variation of this locus suggests its association with gene regions which would be under strong selective pressure against possible mutations. The transferability of locus CRCAc1 to A. angustifolia was first verified by SEBBENN and BitTencourt (2007).

Our results confirmed Mendelian inheritance for the eight microsatellite loci tested and no deviations from expected Mendelian segregation (1:1 or 1:2:1) were detected. In all cases tested (110 in total), the progenies of hand- and open-pollination presented low values for the G-test (maximum of 5.72). Thus, these loci can be used without restriction for studies of the mating system, gene flow, genetic diversity and genetic structure of A. angustifolia. In contrast, BITTENCOURT (2007) detected a significant deviation from the expected 1:1 segregation in open-pollinated progenies of two of the eight sampled seed-trees for loci Ag20 and As90, and in two of the 10 maternal trees for the locus Ag23, in a natural population of A. angustifolia. Nevertheless, in 42 of 52 cases there was no deviation from the expected segregation.

The small number of detected deviations from Mendelian segregation may be the result of sampling error, small progeny sample size, misinterpretation of the size of alleles, or the presence of null alleles (HATTEMER and GILLET, 1989). Genetic factors that cause segregation distortion of significance in molecular markers may be related to pre-zygotic factors that occur between meiosis and gamete fusion and/or post-zygotic factors that occur between fertilization and analysis of the markers. In the first case, three process may occur: (i) meiotic formation occurs with unequal proportions of the types of complementary gametes during meiosis; (ii) selective gametic viability which results in gametic types differing in their ability to survive to fertilization; and (iii) gametic reproductive success which results when gametic types differ in their ability to fertilize the ovules, which, for example, can occur due to gametic self-incompatibility. In the second case, selective mortality related to post-zygotic viability may be occurring between the fertilization stage and the moment we access the genotypes through molecular markers (GILlet and Gregorius, 1992). In this sense, HuFFord and HAMRICK (2003) found the elimination of homozygous embryos resulting from selfing in Platypodium elegans due to inbreeding depression. In the case of the dioecious $A$. angustifolia, the elimination of homozygous seeds may happen due to mating among relatives, causing biparental inbreeding depression.

The eight microsatellite loci used in this study were developed for A. angustifolia by SALGUEIRO et al. (2005) and ScHmidT et al. (2007), except for CRCAc1, developed by SсотT et al. (2003) for A. cunninghamii and As90 developed for A. subulata. These authors, as well as BITTENCOURT (2007) and SANT'ANNA et al. (2013) who used these loci to study populations of $A$. angustifolia, detected no significant deviation from Mendelian segregation in these loci after applying the Bonferroni correction. These results support the idea that the loci used herein are not linked in the chromosomes and segregate independently. Thus, these loci can be used together in genetic analyses that require loci that are not linked, such as analyses of mating system and parentage.

\section{Conclusions}

The eight microsatellite loci analyzed (Ag20, Ag23, Ag45, Aang01, Aang14, Aang28, As90 and CRCAc1) can be used for genetic studies in A. angustifolia, because they present Mendelian segregation in hand- and openpollinated progeny of monoecious and dioecious trees.

\section{Acknowledgments}

M. A. DANner would like to thank the Coordenação de Aperfeiçoamento de Pessoal de Nível Superior (CAPES) for granting her a PhD scholarship at UFPR. F. ZANETTE and A. M. SEBbenN are supported by a CNPq scholarship. The authors are grateful to Dr. EvELYN R. Nimmo for her suggestions and correction of the English in the manuscript.

\section{References}

Anselmini, J. I. and F. Zanette (2012): Polinização controlada em Araucaria angustifolia. Cerne 18: 247-255.

Barbosa, C. E. A., T. Benato, A. L. Cavalheiro and J. M. D. ToREZAN (2009): Diversity of regenerating plants in reforestations with Araucaria angustifolia (Bertol.) O. Kuntze of 12, 22, 35, and 43 years of age in Paraná State, Brazil. Restoration Ecology 17: 60-67.

Bergmann, F., H. H. Gregorius and J. B. LARSEN (1990): Levels of genetic variation in European silver fir (Abies $a l b a)$ - are they related to the species declines? Genetica 82: 1-10.

Bittencourt, J. V. M. (2007): Genetic diversity and dynamics in remnant patches of Araucaria angustifolia forest in Paraná State, Brazil: Implications for conservation and restoration. $228 \mathrm{p}$. Thesis, The University of Reading.

Bittencourt, J. V. M. and A. M. Sebbenn (2007): Patterns of pollen and seeds dispersal in a small, fragmented population of the wind-pollinated tree Araucaria angustifolia in southern Brazil. Heredity 99: 580-591.

Bittencourt, J. V. M. and A. M. SebBenn (2008): Pollen movement within a continuous forest of wind-pollinated Araucaria angustifolia, inferred from paternity and TwoGener analysis. Conservation Genetics 9: 855-868.

Bittencourt, J. V. M. and A. M. SebBenn (2009): Genetic effects of forest fragmentation in high-density Araucaria angustifolia populations in Southern Brazil. Tree Genetics \& Genome 5: 573-582.

Gillet, E. and H. H. HATTEMER (1989): Genetic analysis of isoenzyme phenotypes using single tree progenies. Heredity 63: 135-141.

Gillet, E. and H. H. Gregorius (1992): What can be inferred from open-pollination progenies about the source of observed segregation distortion? A case study in Castanea sativa Mill. Silvae Genetica 41: 82-87.

Guerra, M. P., V. Silveira, M. S. Reis and L. Schneider (2002): Exploração, manejo e conservação da araucária (Araucaria angustifolia). In: Simões, L. L. and C. F. LiNO. Sustentável Mata Atlântica: a exploração de seus recursos florestais. São Paulo: SENAC. p. 85-101. 